\title{
A Ubiquitous Reflective E-Portfolio Architecture
}

\author{
Marcos Forte ${ }^{a, *}$, Wanderley L. de Souza ${ }^{a, b}$, Roseli F. da Silva ${ }^{c}$, Antonio F. do Prado ${ }^{b}$, \\ Jose F. Rodrigues Jr. ${ }^{d}$ \\ a Postgraduate Program in Health Management and Informatics, Federal University of São Paulo, Brazil \\ ${ }^{\mathrm{b}}$ Computer Science Department, Federal University of São Carlos, Brazil \\ c Medicine Department, Federal University of São Carlos, Brazil \\ d Computer Science Department, Institute of Mathematical and Computer Sciences, University of São Paulo, Brazil
}

\section{A R T I C L E I N F O}

\section{Article history:}

Received 7 July 2012

Received in revised form

21 May 2013

Accepted 1 June 2013

Keywords:

Electronic portfolio

Ubiquitous computing

Architecture

Evaluation

User perception

Netbook

\begin{abstract}
A B S T R A C T
Background: In nurse and in medicine courses, the use of reflective portfolios as a pedagogical tool is becoming a common practice; in the last years, this practice has gradually migrated from paper-based to electronic-based portfolios. Current approaches for reflective e-portfolios, however, do not widely operate at outdoor sites, where data networks are limited or nonexistent. Considering that many of the activities related to nurse and medicine courses relate to professional practices conducted in such conditions, these network shortcomings restrict the adoption of e-portfolios.

Purpose: The present study describes the requirements specification, design, implementation, and evaluation of the Ubiquitous Reflective E-Portfolio Architecture, a solution proposed to support the development of systems based on mobile and wired access for both online and offline operation.

Methods: We have implemented a prototype named Professional Practice Module to evaluate the Ubiquitous Reflective E-Portfolio Architecture; the module was based on requirements observed during the professional practice, the paper-based portfolio in use, and related learning meetings in the Medicine Course of a Brazilian University. The evaluation of the system was carried out with a learning group of 2nd year students of the medicine course, who answered to extensive evaluation questionnaires.

Results: The prototype proved to be operational in the activities of the professional practice of the Medicine Course object of the study, including homework tasks, patient care, data sharing, and learning meetings. It also demonstrated to be versatile with respect to the availability of the computer network that, many times, was not accessible. Moreover, the students considered the module useful and easy to use, but pointed out difficulties about the keyboard and the display sizes of the netbook devices, and about their operational system. Lastly, most of the students declared preference for the electronic Professional Practice Module in internal and in group activities, and for the paper-based version while in patient attendance.

Conclusions: There is evidence that the environment where the professional practice takes place influences the usage of the e-portfolio. Mobile devices were able to support students in their professional practice; however, these devices present characteristics that must be judiciously selected, otherwise, they may limit the execution of important tasks. The main
\end{abstract}

\footnotetext{
* Corresponding author. Tel.: +55 115576 4347; fax: +55 1155726601.

E-mail addresses: marcosforte@fsa.br,wm.marcosforte@gmail.com (M. Forte). 
shortcoming identified during the evaluation tests was about the use of the module, and of the access device, during patient attendance. For this reason, we have envisioned a new version of the Professional Practice Module that shall follow a twofold requisite: by one side, it will include all the features of the module, to be used at the university or in the students' homes; from the other side, it will include only the features that are essential for the practice of patient attendance.

(c) 2013 Elsevier Ireland Ltd. All rights reserved.

\section{Introduction}

Over the last decade, an important change has occurred in medical education. The focus of curricula has shifted from the acquisition of knowledge to the achievement of competence [1]. This new scenario has increased the relevance of clinical practice in the learning process and, by consequence, the use of Reflective E-Portfolios. In the literature, there are several proposals for portfolios that support clinical practice [2-7], but often they fail in supporting the multiple characteristics of ubiquitous learning instruments. Some solutions use platform specific languages and frameworks that restrict the number of compatible devices [4,6,7]. Others use Web-based technologies $[2,3,5]$ that restrict the environments where they can be used.

Although it is common to describe mobile Web-based portfolios as ubiquitous, this is only valid when it is guaranteed that the Web server will be accessible through wireless networks. It is easy to meet this condition in controlled environments, such as a hospital or a university campus. But in other situations, such as in external outdoor professional practice - for instance, at suburban residences, this condition cannot be guaranteed. Even with the expansion of cellular networks, the current coverage is not broad enough; it is estimated that 3G networks cover only $45 \%$ of the world population (2011), with much of this coverage concentrated in large cities [8].

In this context, we propose an architecture for the development of ubiquitous reflective e-portfolios that support a diverse scope of devices and platforms, and that can be used independently of data networks. In order to evaluate the proposed architecture, we implemented a prototype called Professional Practice Module (PPM) that was used for a period of five months in practice activities of students and professors of a Medicine Course of a Brazilian University. In particular, this study aims at answering the following research questions:

- RQ1 - What technologies can be used to implement a Ubiquitous Reflective E-Portfolio Architecture that supports professional practice activities in a medicine course?

- RQ2 - What are the users' perceptions about the utility, ease of use, and other aspects related to the Professional Practice Module?

- RQ3 - What were the perceptions of users regarding the use of netbooks as the primary access device?

- RQ4 - How the Professional Practice Module is affected by different learning environments?

\section{Methods}

\subsection{Study scenario}

The scenario of this study is a Medicine Course, established in 2006, of a Brazilian University. The pedagogical program of the course is competences-oriented, following a socioconstructivist educational approach. The course uses its own competence framework, which has been defined through a workshop carried out by the coordination crew of the course. The methodology of the workshop was to have distinguished and experienced medical professionals to reflect about the medical practice. The result of the workshop was the definition of a set of competences considered the most fundamental to achieve excellence in a medical course and, at the same time, in consonance with the Brazilian Curriculum of Undergraduate Medicine [9].

Differently from traditional lecture courses, this course is based on activities organized in three educational units: Education Unit of Simulation of Professional Practice (EUSPP), Education Unit of Professional Practice (EUPP), and Education Unit of Elective Activities (EUEA). As it makes extensive use of a paper-based reflective portfolio of all of the students' activities, its coordinators plan to migrate it to an e-portfolio. In this sense, an initial pilot project was developed comprehending only unit EUSPP; in the present work, we describe a second project that extended and improved the pilot project, and that migrated to unit EUPP. Parallel to this project, an electronic evaluation system will be integrated in the Professional Practice Module so to complete all the required functionalities of the system.

In unit EUPP, the activities take place in real scenarios of professional practice and depend on the supervision of a preceptor - a physician from the local public health system. Professional Practice scenarios aim at developing the student's expertise and his/her autonomy for intervening in real situations; because of their importance, more and more Professional Practice scenarios are presented along the course.

The students assist a pre-determined set of families, usually at the residence of the patients, located in peripheral regions, where data network infrastructure is scarce. These patients are people enrolled in a family health program. Based on patient profiles that are defined by the facilitator, the patients are screened by health agents of the family health units where a given student will be associated. Before the first contact with the patients, the students receive information about their characteristics, and are allowed to access the patients' records.

This patient assistance produces data, which is selected, prepared, and discussed in meetings of small learning groups 
- typically eight students, preceptor, and facilitator. This strategy leads students to get in touch and to share responsibilities with all the parties involved in the process: patients, family members, health care professionals, colleagues, and professors. In the work of Silva and Sá-Chaves [10], there is a detailed description about the reflection process of the students, and about the use of the portfolio as a strategy for personal and professional development.

The teaching-learning process of the Education Unit of Professional Practice is composed of the following stages, all registered in the portfolio [10]:

- Provisory synthesis: The students elaborate clinical histories based on patient care activities; then, the clinical histories are discussed in group sessions; the discussions allow the identification of knowledge gaps, raising alerts about competences that must be acquired or improved; the product of this stage is a set of Learning Questions that will guide the students in the search of new competences;

- Individual synthesis: Each student is responsible for investigating information that relates to the Learning Questions of his/her group;

- New synthesis: Based on the Learning Questions, on the Individual Synthesis, and on group discussions, the students prepare a new synthesis document that carries deep conceptual, methodological, and scientific findings that will fit their learning and practical needs; this final document is then used to elaborate patient care plans.

\subsection{Data collection and participants}

The data collection occurred in three phases (Table 1): Requirements Specification, Prototype Evaluation I, and Prototype Evaluation II. For conducting these phases, first we selected the participants: patients, students, and facilitators from the 1st and 2nd year of the Education Unit of Professional Practice.

In the first phase, we elaborated a detailed record based on data that was gathered during patient attendance observation. We also collected data from two meetings of small learning groups. Concomitantly, three focus group meetings were carried out to exchange experiences between the researcher and the facilitators of the Educational Unit of Professional Practice. After a first analysis of the collected data, we elaborated a structured questionnaire with 10 closed-ended questions. The information collected in this phase was essential to specify the requisites of the proposed architecture and its prototype, and the characteristics of the access device.

In the last two phases, an evaluation of the Professional Practice Module prototype was conducted by volunteers of one small learning group - all the volunteers agreed to exclusively use the prototype. Nine netbooks (ASUS Eeepc model 701) were used for internal and external activities during evaluation phases. Since the evaluations were carried out with a small number of participants, we decided not to use devices with different characteristics; otherwise we would have a large number of variables and, hence, problems in the conclusive interpretation of the results.

In the second phase, two months after the implementation of the prototype, two tasks were carried out: we observed the use of the prototype during two meetings of small learning groups; and we evaluated the success of the implementation via a structured questionnaire. The first part of the questionnaire focused on the characterization of the users; it contained three questions: user gender, user's possession of a personal computer, and type of the user internet access at his/her residence. The second part aimed at delineating their initial impressions and expectations about using the module. It contained questions organized in four groups; G I: questions about computer usage; G II: questions regarding the use of the netbook as an access device; G III: questions related to the perceived ease of use of the PPM - adapted from Davis [11,12]; and G IV: questions related to the perceived usefulness of the PPM - adapted from Davis [11,12].

In the third phase, now five months after the implementation of the prototype, two other tasks were conducted: we observed the use of the prototype during four patient attendances, and we applied a semi-structured questionnaire. In this phase, the facilitator did not take part because the evaluation comprehended the use of the module during patient attendance; an activity that is exclusive to the students. The first part of the questionnaire was composed of three closed questions whose intent was to determine how the module was used in different environments of professional practice; E1: during meetings of small learning groups - in controlled conditions, and with data network support; E2: at the households of the patients - in external, uncontrolled conditions, with limited data network support; and E3: at the students' home - in external conditions, and with data network support. The second part was composed of open questions about the experience with the prototype and with the access device.

\subsection{Ethical issues}

For ethical considerations, the research was approved by the Medical Research Ethics Committee of the university. Before agreeing to voluntarily participate in the study, all participants received written and oral information about the study. Anonymity and confidentiality were guaranteed to participants.

\section{Results}

\subsection{Requirements specification}

In the requirements specification phase, the data gathering from student medical attendance observations, resulted in the following information: average attendance time $-36 \mathrm{~min}$ per patient; list of attendance places - such as backyard, living room, kitchen, garage, and sidewalk; and identification of noise - such as TV, cars, animals, and other people. Furthermore, the students collected descriptive information from each patient: socioeconomic data, disease, symptoms and signs, medications being used, record of exams, lifestyle, habits of physical activity, family relations, and data about employment and income. Students also collected information about their patients through the health agents, preceptors, and health records stored in the Family Health Units. Also, a shortage of wireless computer networks was observed, as most of the patients live in peripheral regions. 
Table 1 - Data collection and participants.

\begin{tabular}{|c|c|c|c|}
\hline Study phase & Context & Participants description $^{a}$ & Data collection method \\
\hline \multirow{4}{*}{ Requirements specification } & Patient attendance & 4 students +8 patients & Observation \\
\hline & Small Learning Group Meeting $(\times 2)$ & 8 students +1 facilitator & Observation \\
\hline & Facilitators meeting $(\times 3)$ & 5 facilitators & Focus group \\
\hline & 2nd-year-students meeting & 37 students & Structured questionnaire \\
\hline Prototype evaluation I & Small Learning Group Meeting & 8 students $^{\mathrm{b}}+1$ facilitator $^{\mathrm{b}}$ & Structured questionnaire \\
\hline \multirow{2}{*}{ Prototype evaluation II } & Patient attendance & 2 students ${ }^{b}+4$ patients & Observation \\
\hline & Small Learning Group & 8 students ${ }^{b}$ & Semi-structured questionnaire \\
\hline
\end{tabular}

Other minor observations contributed to the design as well. We verified that the students wrote down the information in notepads, except for one, who wrote straightly in the paperbased portfolio. Some students had problems in retrieving the information previously stored; and one of the students offered his portfolio so that we could observe usual annotation patterns.

From two meetings of small learning groups, we observed that each student handles a text with information about either his/her activities, or a clinical history. Once in the meeting, each student must provide a hard-copy of the text to all members of the group. In three meetings with facilitators, we discussed the design of the Professional Practice Module and, so, we could identify additional features to supplement its functionalities. One of our findings was the need to collect data for epidemiological research.

In the structured questionnaire, we complement the information obtained in the observations, among which the following stand out: average number of patients per student of 5.12 with a standard deviation of 1.52, average attendance time per period - morning or evening $-2 \mathrm{~h}$ and $45 \mathrm{~min}$ with a standard deviation of $1 \mathrm{~h}$ and $6 \mathrm{~min}, 50 \%$ of the students moving to a service location walking or by public transportation, and the others moving by car.

\subsubsection{Mobile access devices specification}

Based on requirements specification we considered the following aspects for choosing the most appropriate device:

- Data Entry - for data input in the portfolio, the students generally use free text. Therefore, devices with no keyboard or with small sized keyboards, would impact the students' motivation to use the application. In fact, according to [13], limited keyboards demand three times as much time for text typing;

- Screen size - during the meetings of small learning groups, the members read, on average, eight pages of text; besides that, generally, the attendance sites are dimly lit for electronic reading. Hence, small screens would limit reading activities;

- Weight and size of the device - nearly $50 \%$ of the students have to walk or depend on public transportation to get to the Health Units and to the houses of the patients;
- Battery life-based on the average attendance time per period, the device should operate at least four hours on battery.

Based on our market survey and on the aspects of the application, a cost-benefit analysis indicated, among access devices available in Brazil, that the netbook ASUS model 701 was the best choice. This device is configured with a 7 inch screen, has a keyboard with $82 \%$ the size of a desktop keyboard, weighs $910 \mathrm{~g}$, has the size of a regular textbook, operates with major Web browsers, and costs, in Brazil, less than half the price of a smartphone.

\subsection{Ubiquitous Reflective E-Portfolio Architecture}

The creation of applications for ubiquitous environments has two challenges: adapting the interface and functionality of the application to access devices with different characteristics, and enabling the use of the application regardless of the existing data network. Considering the context of use activities related to the learning process, there is one more challenge: working with different types of content (e.g. documents, images, audio, and video). So, in order to facilitate the development, we designed an architecture that provides solutions for these challenges, helping developers focus on the learning design, rather than on the technological issues. To widen the scope of use of the proposed solution, the architecture does not adhere to any predefined information model [3] or competence framework [2], allowing that it be specialized for different learning contexts and areas of knowledge.

Fig. 1 shows the architecture as designed to suit the characteristics of the Ubiquitous Reflective E-Portfolio. The architecture is composed of the following elements: Ubiquitous Reflective E-Portfolio client, to be installed on the access device; Ubiquitous Reflective E-Portfolio server; and Content Application Server (CAS), which is used to store and to index the content of the Reflective E-Portfolio.

The Ubiquitous Reflective E-Portfolio Architecture is based in the design pattern Model-View-Controller (MVC) [14]. This design pattern offers a diversity of presentation (View) layers that, although specific to each access device, do not demand alterations at layers Model or Control, which become universal to the project. Another characteristic of the project was the possibility of using access devices with different configurations - such as size and resolution. In order to have the 


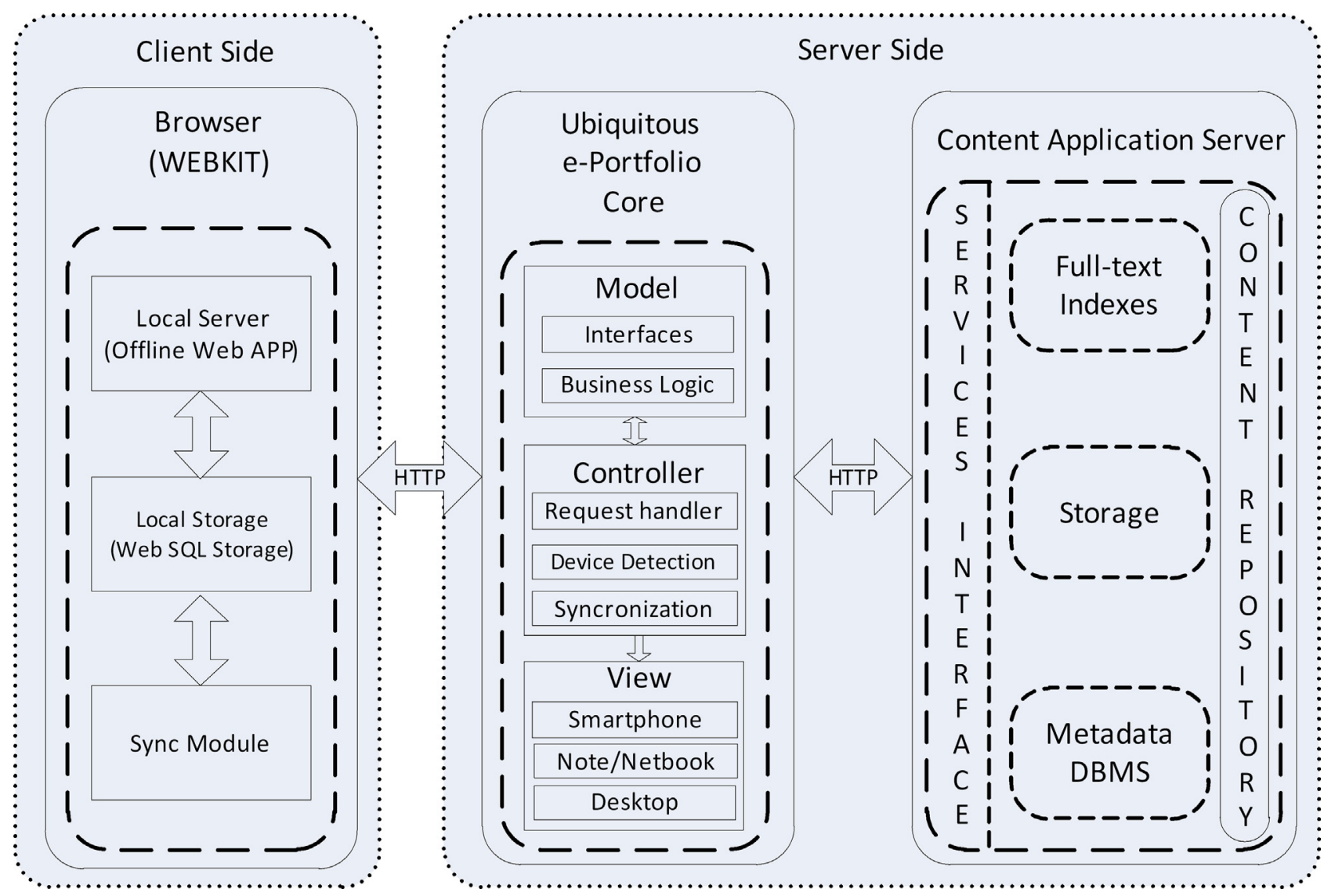

Fig. 1 - Ubiquitous Reflective E-Portfolio Architecture.

proposed architecture satisfy this requirement, we integrated to it a specific component, named Device Detection, that is dedicated to the identification of the type of access device that issued the requisition to the server. This information then, enables the architecture to select the view layer that will produce the best visualization and interaction according to the characteristics of the access device (e.g. smartphone with touchscreen or note/netbook with a mouse). Furthermore, the view layers use Responsive Web Design methodologies and CSS3 Media Queries [15]; techniques that support client-side adaptations of the layout and of the components of the page (e.g. resizing of images and videos), allowing a fine adjustment of the viewing on devices of the same category (e.g. smartphones with different resolutions and screen size).

Another problem in designing the architecture was to define the storage requirements in conformity with the great variety of content of the portfolios. In other words, an eportfolio combines documents (e.g. narratives, and research material) and data-centered (e.g. patient data) approaches [16]. A common alternative for storage is the use of relational databases; however, current approaches based on this technology are limited in respect to the diversity of file formats and, due to the lack of a wide-accepted standard. Another alternative is to use a file system directory structure to create a hierarchy of documents [16], but this approach does not have native support for indexing and versioning. For these reasons, the architecture was conceived with alternative solutions: the Content Repository API for Java (JCR) [17] and the Content Management Interoperability Services (CMIS) [18]. These components enable interoperability between systems, providing indexing, versioning, and metadata storage.

\subsubsection{Offline support}

Due to the mobility of the students, especially in regions and in circumstances where data networks are not available, server access is notably limited. Thus, it was a requirement that the architecture foresaw the possibility, even offline, of retrieving the records of the patients, and also that it permitted the inclusion of new data for later synchronization. This functionality is provided by the client-side components, as observed in Fig. 1: the Local Server emulates a web server so that, if a data network is not detected, the Local Storage provides storage and retrieval from a local database; the Sync Module, in turn, synchronizes the information recorded in the local database with the remote database (server-side), allowing the user to access updated information on multiple devices, what improves the reliability of the storage system. The problem about two databases is that it can generate consistency problems in case the synchronization process is not appropriate [19]. In order to avoid such issues, the proposed architecture provides components for data synchronization 


\subsection{Professional Practice Module implementation}

As already mentioned, we have implemented a specialization of the proposed architecture - the Professional Practice Module (PPM) - designed to support the needs of the Educational Unit of Professional Practice. The module was developed throughout a six-month period with support of server technologies Java Server Faces 1.2, Java Persistence API, Tomcat 6, and content manager Alfresco CE 3.3 [20]. The client-side of the module counted on technologies CSS, Javascript, Jquery, and HTML5.

Fig. 2 illustrates the relationship between the activities of the student's reflection, described in Section 2.1, and the main components used in the Professional Practice Module. The module supports individual activities (1), (2), (4), which often occur outside the campus, and group activities (3), (5) that occur in the campus.

Considering that the Educational Unit of Professional Practice relates to patient care, and to ways of providing a better organization of the collected information, hence, the Patient Care component is a composition of several subcomponents. Some subcomponents are Web forms related to family health public programs, and to common health care data (e.g. blood pressure); other subcomponents allow free text insertion being associated with four kinds of patient documentation: Clinical History and Examination, Life History, Health Needs, and Plan of Care. And, also, tools to search for drug references, to consult the International Classification of Diseases (ICD-10), and to calculate the Body Mass Index (BMI). These tools were developed, specifically for the module, using the components of the Ubiquitous Reflective E-Portfolio Architecture, enabling the use of such tools even offline.

Fig. 3 illustrates the Life History component interface, which allows for retrieving and registering the patient's life history, as well as for writing narrative descriptions. In the figure, the interface is marked from (1) through (4); beneath the menu (1), there are two areas for text edition: the first one - marked (2) - is used for specific notes about the selected patient (e.g. José Silva), and the second one, the User Editor - marked (4) - is used for making annotations that are independent of specific patients, such as narratives and syntheses. Students use the first editor to recover patient-related information, and the second editor to take notes on eventual reflections to be used, later on, during the meetings of small learning groups.

The text created in the second editor (4) is versionmanaged through the controls presented in its respective panel - marked (3) in Fig. 3. This versioning management permits that any text edition be time and date registered without overwriting previous versions, ensuring that modified documents are new documents in the history of documents [16]. With this functionality, student and facilitator can monitor the elaboration and the revision of textual information, optimizing the text-based reflection and evaluation processes. Another feature of the second editor is the possibility of using tags to label keywords - this feature is important because it allows for tracking epidemiological data, and because it assists the facilitator that can come up with lists of keywords extracted from the students' narratives. The Professional Practice Module also supports collaborative work among the students, who can share text and research information.

We also conducted laboratory tests with alternative access devices (notebooks, desktops, iPhone, and Android devices) aiming at evaluating the potential of adaptation of the architecture with other systems. Although we observed the need for some adjustments in the interface, the Device Detection subsystem worked properly.

\subsection{Prototype evaluation I: structured questionnaire}

The first part of the questionnaire was filled out by six women, including the facilitator; and three men. All the participants had personal computers - seven notebooks and two desktops; and all of them had broadband Internet at home.

The results of the second part of the questionnaire are presented in Table 2. Due to the variability and psychological bias of the groups, we evaluated the internal data consistency using Cronbach's alpha coefficient, with a 95\% lower confidence limit. Except for group of questions (GQ) I, which was discarded, all the groups of questions demonstrated to be reliably consistent in respect to an overall proposition for each group - as stated in column GQ of Table 2. Since our experiments are long-term, and have a limited number of access devices, we were not able to use a large number of participants. However, we can still verify the representativeness of our statistics by evaluating their statistical power (column Power of Table 2) considering the average of each group, which is calculated for a confidence significance level of 0.05 , and assuming the same standard deviation for the null hypothesis. For all of the groups the power is higher than 0.8 and, thus, representative according to Mazen et al. [21].

\subsection{Prototype evaluation II: semi-structured questionnaire - part I}

The results of the questionnaire (Q1-Q3) indicate that the use of the Professional Practice Module took place, mainly, in two contexts of use: during small learning group activities (I), when information sharing was an important issue; and in the residences of the students (III), where the main tasks were to type the information collected during the visits to the patients, and to elaborate the narratives and/or synthesis. The detailed results of this part are available in Appendix A.

Supplementary material related to this article can be found online at http://dx.doi.org/10.1016/j.ijmedinf.2013.06.005.

\subsection{Prototype evaluation II: semi-structured questionnaire - part II}

The results of the questionnaire (Q4-Q11) are summarized in Table 3. We computed the results of this part using the qualitative content-based analysis proposed by Bardin [22]. For example, $62.5 \%$ of the answers given to question 4 were related to the netbook input and output characteristics - in this context, $40 \%$ of the answers reported that the size of the keyboard was small, $40 \%$ of the answers reported that the size of the keyboard was good, and $20 \%$ of the answers did not cite the keyboard. 


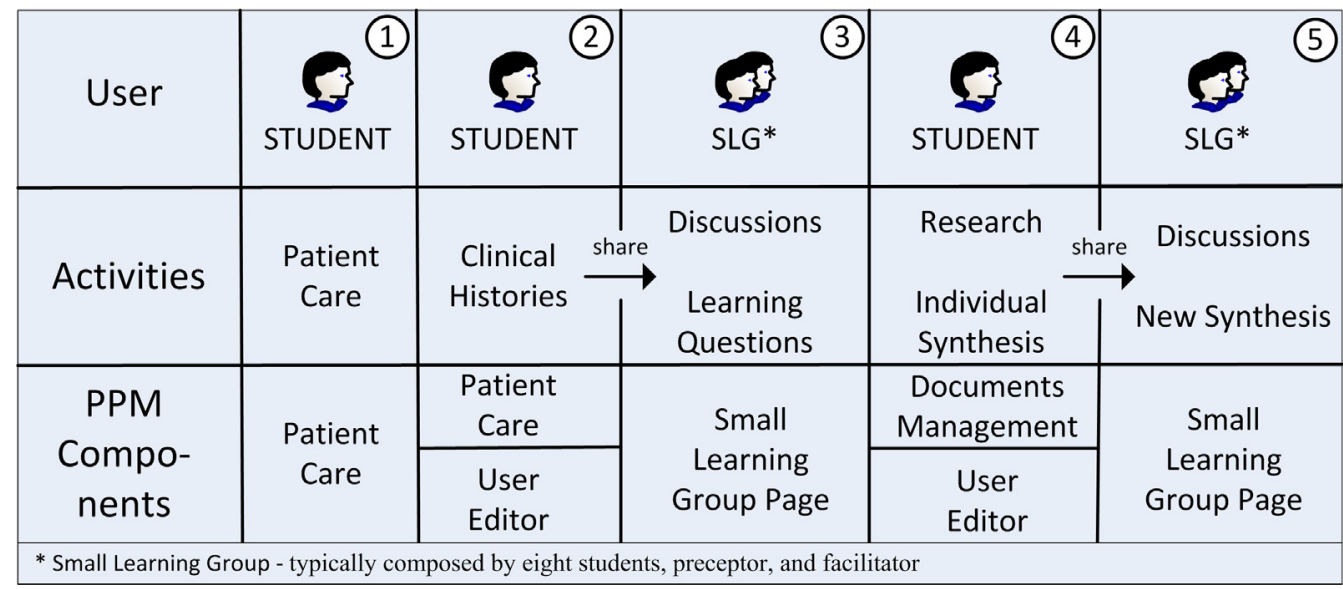

Fig. 2 - Professional Practice Module components and related activities.

\section{Discussion and conclusions}

Following we analyze the data gathered through the evaluation questionnaires of the Ubiquitous Reflective E-Portfolio prototype. The analysis goes according to the research questions proposed in Section 1, which we discuss one by one as we refer to the results obtained during the two phases of the prototype evaluation.

RQ1 - What technologies can be used to implement a Ubiquitous Reflective E-Portfolio Architecture that supports professional practice activities in a medicine course?

Based on the results of the requirements phase, we observed two important characteristics: first, the learning environments are very heterogeneous, ranging from controlled environments - such as the university campus, where the activities are structured and organized, and the students are usually sitting around a table, to uncontrolled environments - such as the patients' homes, where the attendances can occur with students standing, with distracting factors (e.g. sound noise, pets), and without access to a data network; second, the demand for data entry/view, and the need for portability of the access devices, vary greatly during the various stages of the teaching-learning process.

Supporting operation without a data network access is an issue also reported in the work of Horner [23], who describes an offline solution based on a Web Server set locally at the client device; in this scheme, the local server responds when requisitions are issued by the client application, logging them for later synchronization with the remote Web Server. Moore et al. [24] describe another solution, but based on the iPhone. The drawbacks of both solutions lie in the fact that the local Web server can consume the scarce resources of the access device, it cannot be implemented in devices that restrict the installation of certain applications-as the iPhone, and are generally incompatible with operating system updates.

Differently, in this work, the problem of using the EPortfolio offline is treated with emergent broadly accepted Web standards, the HTML5. This way, in order to have the Professional Practice Module working on mobile devices, it is only necessary that its Web browser be compatible with standard software WebKit [25]. Currently, this condition is fulfilled by any tablet or smartphone device that uses operating systems Android, IOS, or WebOS; for notebooks or desktops, Chrome and Safari are suitable browsers. Like any new technology, the HTML5 has some problems as the lack of some implementation of functionalities and lower performance, mainly in mobile devices, but these problems are diminishing rapidly with the new generation of browsers.

To support the use of different devices in different usage context, the architecture provides device detection and database synchronization components, allowing the user to use a notebook/desktop in activities that require a greater amount of typing and/or data visualization, or a smartphone when portability is more important.

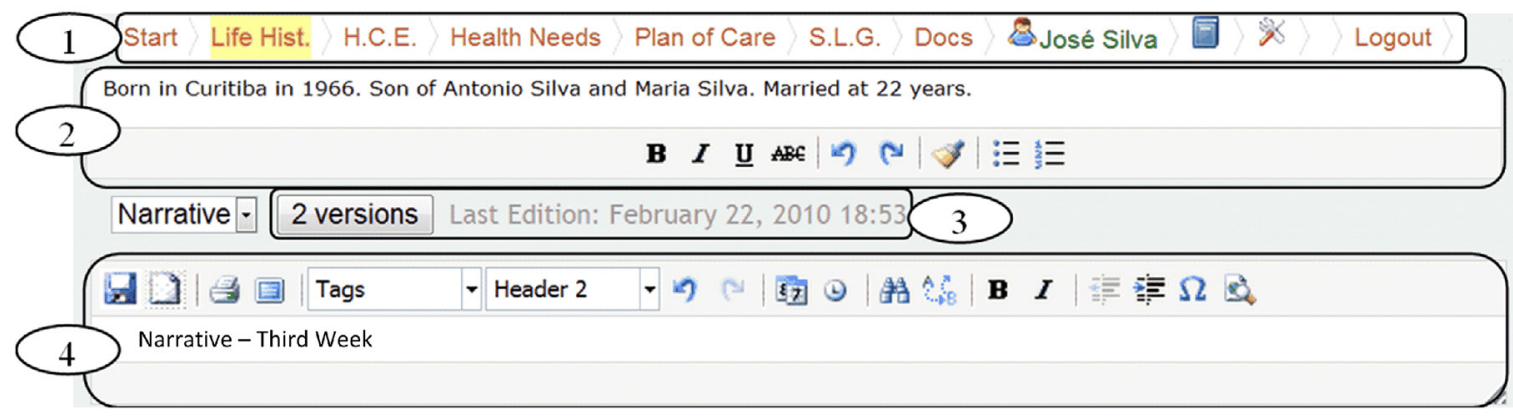

Fig. 3 - Professional Practice Module textual input interface. 
Table 2 - Descriptive statistics - prototype evaluation I: structured questionnaire - part II.

\begin{tabular}{|c|c|c|c|c|c|}
\hline GQ & Item & Mean $^{a}$ & SD & Alpha & Power \\
\hline \multirow{4}{*}{$\begin{array}{l}\text { II-Use of the netbook } \\
\text { as an access device }\end{array}$} & $\begin{array}{l}\text { Q3 - It is easy to carry the netbook for my } \\
\text { professional practice activities. }\end{array}$ & 5.33 & 1.22 & \multirow{4}{*}{0.91} & \multirow{4}{*}{0.97} \\
\hline & $\begin{array}{l}\text { Q4 - I can easily type using the keyboard } \\
\text { of the netbook. }\end{array}$ & 3.67 & 1.32 & & \\
\hline & $\begin{array}{l}\text { Q5 - Despite the small size of the netbook } \\
\text { screen, I can properly read the text and } \\
\text { the web pages. }\end{array}$ & 4.89 & 1.54 & & \\
\hline & $\begin{array}{l}\text { Q6 - I consider the netbook interface } \\
\text { simple and easy to use. }\end{array}$ & 4.11 & 2.15 & & \\
\hline \multirow{5}{*}{$\begin{array}{l}\text { III - Perceived ease of } \\
\text { use of the PPM }\end{array}$} & $\begin{array}{l}\text { Q7 - It would be easy for me to learn how } \\
\text { to operate the PPM. }\end{array}$ & 5.00 & 1.22 & \multirow{5}{*}{0.93} & \multirow{5}{*}{0.99} \\
\hline & $\begin{array}{l}\text { Q8 - I would find it easy to use the PPM to } \\
\text { do what I want it to do. }\end{array}$ & 5.00 & 1.50 & & \\
\hline & $\begin{array}{l}\text { Q9 - My interaction with the PPM would } \\
\text { be clear and understandable. }\end{array}$ & 5.22 & 1.56 & & \\
\hline & $\begin{array}{l}\text { Q10 - It would be easy for me to become } \\
\text { skillful at using the PPM. }\end{array}$ & 5.44 & 1.42 & & \\
\hline & $\begin{array}{l}\text { Q11 - I think that the PPM will be easy to } \\
\text { use. }\end{array}$ & 5.11 & 0.93 & & \\
\hline \multirow{6}{*}{$\begin{array}{l}\text { IV - Perceived } \\
\text { usefulness of the } \\
\text { PPM }\end{array}$} & $\begin{array}{l}\text { Q12 - Using the PPM would allow me to } \\
\text { accomplish tasks more quickly. }\end{array}$ & 5.11 & 1.27 & \multirow{6}{*}{0.97} & \multirow{6}{*}{1.00} \\
\hline & $\begin{array}{l}\text { Q13 - Using the PPM would improve my } \\
\text { performance in the EUPP activities. }\end{array}$ & 4.67 & 1.22 & & \\
\hline & $\begin{array}{l}\text { Q14 - Using the PPM would increase my } \\
\text { productivity. }\end{array}$ & 5.11 & 1.45 & & \\
\hline & $\begin{array}{l}\text { Q15 - Using the PPM would increase my } \\
\text { effectiveness in the EUPP activities. }\end{array}$ & 4.89 & 1.27 & & \\
\hline & $\begin{array}{l}\text { Q16 - Using the PPM would make it easier } \\
\text { to carry out my EUPP activities. }\end{array}$ & 4.89 & 1.27 & & \\
\hline & $\begin{array}{l}\text { Q17 - I believe that the PPM will be useful } \\
\text { in the practice of my EUPP activities. }\end{array}$ & 5.56 & 1.13 & & \\
\hline
\end{tabular}

According to the evaluation questionnaires, the design and implementation choices proved to be valid, since the students were able to use the prototype in the learning environments of the Education Unit of Professional Practice, including the external environments without data network.

Overall, the proposed architecture is generic, not being limited to the educational context; it could be used to simplify the development of applications that demand multiple access devices to work even in the absence of an active data network.

RQ2 - What are the users' perceptions about the utility, ease of use, and other aspects related to the Professional Practice Module?

The prototype evaluation showed that the users considered the Professional Practice Module easy to use and helpful. As positive aspects, the users mentioned: it helps in the organization of information; it facilitates the information exchange among members of a small learning group; and it contains useful tools to support professional practice as, for example, drug references. And, as negative aspects: adaptation and operation shortcomings; data network failures during the meetings of small learning groups; doubts about the reliability of the information storage; insufficient training; and difficult to maintain eye contact with the patient when using the netbook.

The users suggested several modifications to the interface and the addition of new features to the Professional Practice
Module. Most of the suggestions referred to the text editors; more commonly, the demand was for a better control of the text font in order to improve readability. Some general suggestions were also detected: better execution efficiency; inclusion of a report module so as to simplify the task of printing patient data; and an automatic system to inform the date of the last update of the patient data. The students also mentioned that, during the professional practice, they had to change the family health unit they were working on and, consequently, had to work with a new set of patients. This fact retarded the progress of the professional practice, what demanded an extra time for the students to get used with the Professional Practice Module. It is possible, hence, that this shorter time availability might have been critical in the decision of some students to return to the paper-based portfolios. This speculation is corroborated by Kay [26], who found that undergraduate students enrolled in a program that uses mobile computers, were happier, less anxious, and less angry as their knowledge in the use of computers increased over time.

Also relevant is the fact that some students demonstrated a great apprehension about safely using the Professional Practice Module. Specifically, they mentioned the possibility of having the equipment stolen, or the possibility of data loss. We perceived that such apprehension decreased with time because, during the evaluation period, we did not register any case of stolen equipment; and because the cases of data loss 
Question

4 - Describe your experience with the screen, keyboard, operating system, and other factors.

5 - In addition to the Professional Practice Module, which of the other applications available in your netbook did you use?

6 - Do you have any constraints on using the netbook for attending patients? Discuss your answer. netbook hardware -

Context units

Netbook Data Entry/View (62.5\%).

Netbook OS (75\%)

Netbook use adaptation (37.5\%)

Portability $(25 \%)$

Applications used in addition to the PPM $(100 \%)$

Have Reservations? (100\%)

Affects student-patient relationship (50\%)

Reliability (25\%)

General (37.5\%)

7 - What changes do you suggest to the Professional Practice Module?

Text Editors (62.5\%)

Positive Facts $(100 \%)$

8 - Evaluate your experience using the Professional Practice Module, highlighting the positive and negative factors?

9 - During the next semester, will you prefer to work with the electronic version or to return to the paper version of the portfolio? Discuss your answer.

10 - What Internet tools do you use in your academic activities, including the exchange of information between the members of your small learning group?

11 - Do you have any further comments or suggestions?

During Small Learning Group Meetings $(100 \%)$
Registry units

Good keyboard size (40\%)

Small keyboard size $(40 \%)$

Good screen size (40\%)

Small screen size $(60 \%)$

Small font size $(20 \%)$

Difficult to use (100\%)

Prefer windows $(50 \%)$

Could not adapt (33\%)

Adaptation demands time (66\%)

Extra weight bothers (50\%)

Easy to carry (50\%)

Web browser $(100 \%)$

Office Suite (OpenOffice) (50\%)

Video calling (Skype) (25\%)

Multimedia Player (SMPlayer) (25\%)

PDF Reader (Acrobat) (12.5\%)

File Manager (Xandros-explorer) (12.5\%)

Yes $(75 \%)$

No $(25 \%)$

Typing decreases attention (75\%)

The patient may feel uncomfortable (50\%)

Afraid of having the netbook stolen (50\%)

Afraid of losing data (50\%)

Add a reporting module (33.3\%)

Change the interface icons $(33.3 \%)$

Faster execution (33.3\%)

Log last update of the patient (33.3\%)

Alter font size for better visualization (40\%)

Automatic text correction $(20 \%)$

Better identification of text editors $(20 \%)$

Add a text editor for elaborating drafts $(20 \%)$

Better organization of information (50\%)

Ease of doc sharing between SLG (37.5\%)

Support tools (e.g. CID search) (12.5\%)

Difficulties in the module operation (25\%)

Wireless network failures (25\%)

Decreases attention to the patient (12.5\%)

Insufficient training (12.5\%)

Doubts about data storage reliability (12.5\%)

Alternation of patients (12.5\%)

Prefer to use the paper portfolio (62.5\%)

Prefer to use the electronic portfolio (12.5\%)

Prefer to use both (12.5\%)

Undecided (12.5\%)

Prefer to use the paper portfolio (12.5\%)

Prefer to use the electronic portfolio (62.5\%)

Prefer to use both $(12.5 \%)$

Undecided (12.5\%)

Email (100\%)

Search engine $(100 \%)$

Google DOCS (37.5\%)

Medical databases (25\%)

Social networks (12.5\%)

Instant messaging (12.5\%)

Bigger keyboard and Windows (12.5\%)

More training (12.5\%)

Know the results of the evaluations (12.5\%) 
were related to initial usage issues. The data stored in the module databases are encrypted and the access is password protected; in the event of data loss, the student can retrieve them from the backups that are stored on the server, and optionally in pendrives.

Regarding the facilitator's assessment, relevant observations include the simpler and easier access to the students' narratives, and the smaller amount of paper. These results were similar to those obtained by Driessen et al. [27], who report that the tutor considered it to be easier and faster to search and read narratives on the computer. The facilitator also noted that the module collaborates with the development of clinical practice, because its components follow the same steps of clinical reasoning; that is, for 1st and 2nd-year students: medical history; physical examination; identification of problems and health needs; and plan of care. So, when students go into patient care, they can detail each step in the portfolio, which became more efficient in its electronic version due to functionalities of organization, edition, and retrieval of information. In this format, the portfolio is reviewed by the facilitator which gives feedback to the student about his/her achievements.

RQ3 - What were the perceptions of users regarding the use of netbooks as the primary access device?

As we did not find literature about the use of e-portfolios over netbooks, we formulated several questions concerning this issue in stage one of the evaluation. The results demonstrated that the keyboard and the interface of the netbook had the smallest usability ratios. We believe these facts are due to the short time of adaptation, and to the large volume of text that the students have to type on a reduced-size keyboard.

Our investigations suggest that, despite the netbook's greater capacity for input and output - if compared to PDAs and smartphones, it is a challenge to bring together efficient data entry and adequate data presentation in mobile settings; a problem that becomes even worse in the context of the Reflective Portfolio. In fact, we verified that the small keyboard discouraged the use of the netbook for patient attendance. In a similar work, Garret and Jackson [28] observed that the students preferred to type the information that they had collected when they were at home via their own equipment. In another work, Cotterill et al. [29] concluded that there exists preference for paper-based portfolios during activities that demand clinical skills. Horner [23] observed that despite the perceived advantages of e-portfolios, most students chose to continue with the paper-based portfolios. Also according to this work, the main reason for such preference was the short duration of the modules of the respective courses, what did not assure enough time for the students to adapt.

Regardless of the fact that the architecture of the Reflective E-Portfolio supports smartphones, the restrictions of these devices, regarding data input and interface, prevent their use in some student activities. Yet, such devices can increase the ubiquity of the Reflective E-Portfolio for tasks that do not demand large amounts of data, such as querying the patient information, and registering small attendance observations.

During the second evaluation, we observed aspects that reinforce the negative perceptions about the netbook. Besides the sizes of the keyboard and of the display, the respondents reported lack of experience with the Linux Operating System.
The shortcomings with the OS were more apparent when operations, other than using the Professional Practice Module, were being performed, such as printing and configuring the network. Using the module was not an issue because it is web-based and, thus, it is independent of OS. Still, the OS caused problems for the users to adapt to the netbook; in fact, some students decided to use their own notebooks to execute some Windows-only applications. Nevertheless, the students reported that the size and the weight of their notebooks were not adequate for outside activities. In a related study, Garrett and Jackson [28] report that, in using their portable devices, students tend to use other applications - besides the e-portfolio - for all their regular activities.

The equipment used in the evaluation was the model that introduced a new computer category in the market, the netbooks; but the market has not had good acceptance of netbooks with 7 inch display and Linux, and these models have been discontinued. Thus we consider that the use of netbooks cannot be discarded due to the results obtained in this evaluation because the current generation of netbooks has larger keyboards and screens, with more efficient power consumption, and with operating system Windows, what can improve the usability in this category of access device.

RQ4 - How the Professional Practice Module is affected by different learning environments?

The students used the Professional Practice Module in three learning environments: during meetings of small learning groups, during patient attendance, and during individual research - at the university or at home.

The ability of sharing files among the members in a small learning group was the major attraction for the users. The sharing of files was also important in monitoring and evaluating the students; it allowed facilitators and preceptors to retrieve up-to-date information about their students' portfolios. In a study with e-portfolios, reported by Driessen et al. [27], the students considered that a notorious advantage of this technology is the simplicity in sharing information among the members of the group.

The second evaluation of the prototype revealed that the majority of the students claimed to have reservations about the Professional Practice Module because it may affect the student-patient relationship. The students mentioned two reasons: it was difficult to simultaneously type and talk to the patients; and using high-tech equipment in humble dwellings may raise barriers in the relationship with the patient. In order to analyze these reasons, one must take into consideration the little experience of the students both in the use of the module and in the attendance of patients. In this aspect, Alsos et al. [30] found that three factors affect the communication between doctor and patient: (1) the physical form of the access device; (2) the user interface of the application, and (3) the physicians communication practice. Bogossian et al. [4] observed that students are reluctant to use handhelds in front of patients fearing that they would not be viewed as proficient in the use of this device.

During patient attendance observations, we noted that the text editor for input of patient data was little used during attendance activities; instead, the students preferred to store all information in a single text area and, later, reorganize the text at home. We also observed that the features the most used 
were the ones that demanded less typing, and the ones with typing assistants - as autocomplete.

The second evaluation of the prototype also reported that the different learning environments influenced the students' decision about continuing with the Professional Practice Module or returning to the paper-based portfolio. By computing the answers, we verified that the very respondents defined two possible environments of use: during meetings of small learning groups, and during patient attendance. For use during the meetings, $62.5 \%$ of the respondents preferred the Professional Practice Module and $12.5 \%$ preferred the paperbased portfolio. Meanwhile, for use during patient attendance, this tendency is nearly the inverse. Furthermore, $12.5 \%$ of the respondents opted for the joint use of the electronic and paper-based portfolios, and another $12.5 \%$ did not opt, claiming not to have had enough time to evaluate.

\section{Final considerations and conclusions}

This paper presented the Ubiquitous Reflective E-Portfolio Architecture together with the evaluation of the Professional Practice Module, a specialization of this architecture, as part of the practice-based program of the Medicine Course of a Brazilian University. We described a complete experience on the use of mobile web-based technologies for high-level education. In accordance with the latest tendencies, we have put into practice technologies that, although relatively recent, have grown rapidly due to the availability and to the evolution of mobile devices [31].

We found, along our work, that mobile devices could support students in their professional practice; however, these devices present characteristics that must be judiciously selected, otherwise, they may be limited in performing important tasks. Among such features, we mention screen, keyboard, battery life, operating system, and human-computer interface - all of those representing key aspects for the practice.

The evaluation of our work revealed that most students prefer to use the Professional Practice Module in group activities, mainly due to its information sharing functionality. However, the students have demonstrated reluctance for using it during patient care attendance; they have argued that the module could interfere in the relationship with the patients. Some of them even preferred to continue with the paper-based portfolio and, later, type the collected information into the Professional Practice Module.

A natural factor in proposing and introducing a technology that comes along a novel paradigm is its learning curve. Henceforth, we believe that some of the problems observed in the use of the Professional Practice Module were biased by the fact that the students had never worked with the Reflective E-Portfolio and, neither, had enough time for training. Therefore, it is our contention that new evaluations of the module may draw additional conclusions about its use.

Addressing the findings of this work, a new version of the Professional Practice Module is to be developed; this version will consider the results presented in Section 4, including an improved context-aware interface. The design of this interface will answer for the main shortcoming that we identified
Summary points

What is already known:

- The use of paper-based portfolios is migrating to electronic portfolios.

- Most solutions available of electronic portfolios depend on a data network.

- The Professional Practice of students can occur internally or externally to the university campus.

What this study added to our knowledge:

- This study demonstrates that is possible, and how it is possible, to develop and employ an electronic portfolio at a medicine course. The constraints of such a problem included aspects of locality, data network structure, and choice of Web technologies.

- Interference in the relationship with the patients was the main cause of student reluctance in using the electronic portfolio during patient attendance.

- Prototyping and evaluation over netbooks as the choice for employing the electronic portfolio.

during the evaluation tests, that is, the problems of using the module during patient attendance. Thus, the new design will be twofold: by one side, it will include all the features of the module, to be used at the university or in the students' homes; from the other side, it will include only the features that are essential for the practice of patient attendance.

\section{Author contributions}

Marcos Forte: conceptualized and designed the study; designed the main concepts of the proposed architecture; prototyped the implementation; analysis and interpretation of data; writing and revising the article; final approval of the version to be submitted.

Wanderley L. de Souza: assisted with the design of the study; contributed to the development of the proposed architecture; writing and revising the article; final approval of the version to be submitted.

Roseli F. da Silva: contributed with requirements specification; elaborated parts of questionnaires; assisted with the qualitative content-based analysis; writing and revising the article; final approval of the version to be submitted.

Antonio F. do Prado: assisted in the choice of technologies, contributed with the prototyping; revising of the article; final approval of the version to be submitted.

Jose F. Rodrigues Jr.: assisted in analysis and interpretation of data; contributed in data collection; writing and revising the article; final approval of the version to be submitted.

\section{Conflicts of interest}

The authors have no conflicts of interest regarding this study. 


\section{Acknowledgements}

Our thanks to CNPq for sponsoring our research in the context of the INCT-MACC. We also thank Prof. Cibele Russo for her valuable statistical advising.

\section{REFERE NCES}

[1] H.K. Rabinowitz, D. Babbott, S. Bastacky, J.M. Pascoe, K.K. Patel, K.L. Pye, J. Rodak, K.J. Veit, D.L. Wood, Innovative approaches to educating medical students for practice in a changing health care environment: the National UME-21 Project, Acad. Med. 76 (June (6)) (2001) 587-597.

[2] B.M. Garrett, M. MacPhee, C. Jackson, Evaluation of an eportfolio for the assessment of clinical competence in a baccalaureate nursing program, Nurse Educ. Today (2012) [Epub 2012 Jul 10].

[3] P. Staccini, E. Hergon, M. Joubert, M. Fieschi, Collaborative and workflow-oriented digital portfolio: creating a web-based tool to support a nationwide program of practices evaluation in the blood transfusion area, Int. J. Med. Inf. 76 (May-June (5-6)) (2007) 383-392.

[4] F.E. Bogossian, S.E.M. Kellett, B. Mason, The use of tablet PCs to access an electronic portfolio in the clinical setting: a pilot study using undergraduate nursing students, Nurse Educ. Today 29 (February (2)) (2009) 246-253.

[5] J. Pincombe, L. McKellar, M. Weise, E. Grinter, G. Beresford, ePortfolio in Midwifery practice: the way of the future, Women Birth 23 (September (3)) (2010) 94-102.

[6] C. Dearnley, J. Haigh, J. Fairhall, Using mobile technologies for assessment and learning in practice settings: a case study, Nurse Educ. Pract. 8 (May (3)) (2008) 197-204.

[7] S.L. Ranson, J. Boothby, P.E. Mazmanian, A. Alvanzo, Use of personal digital assistants (PDAs) in reflection on learning and practice, J. Contin. Educ. Health Prof. 27 (Fall (4)) (2007) 227-233.

[8] International Telecommunication Union, The world in 2011: ICT facts and figures, 2011 [cited 2013 Jan 2]. Available from: http://goo.gl/vHf7K

[9] A.G. Aquilante, R.F.da Silva, M.B.B.de Souza, R.G.B. Kishi, Implementation of competency-based curriculum in medical education: perspective of different roles, ISRN Educ. 2012 (2012) 7, http://dx.doi.org/10.5402/2012/651426.

[10] R.F.da Silva, I. Sá-Chaves, Reflective training: teachers' representations regarding the use of a reflective portfolio for physician and nurse training, Interface - Comunicação, Saúde, Educação., 2008, 4.

[11] F.D. Davis, A technology acceptance model for empirically testing new end-user information systems: theory and results, MIT Sloan School of Management, Cambridge (MA), 1986 (Doctoral Dissertation).

[12] F.D. Davis, Perceived usefulness, perceived ease of use, and user acceptance of information technology, MIS Quart. 13 (September (3)) (1989) 319-340.

[13] E. Rukzio, C. Noda, A. De Luca, J. Hamard, F. Coskun, Automatic form filling on mobile devices, Pervasive Mob. Comput. 4 (April (2)) (2008) 161-181.
[14] A. Leff, J.T. Rayfield, Web-application development using the model/view/controller design pattern, in: Proceedings of the 5th IEEE International Conference on Enterprise Distributed Object Computing, IEEE Computer Society, Washington, DC, 2001, p. 118.

[15] E. Marcotte, Responsive Web Design, 1st ed., A Book Apart, New York, 2011.

[16] C. Lovis, R.H. Baud, P. Planche, Power of expression in the electronic patient record: structured data or narrative text? Int. J. Med. Inf. 58-59 (September) (2000) 101-110.

[17] Java Community Process (JCP), JSR 283: Content Repository for Java $^{\mathrm{TM}}$ Technology API Version 2.0, 2009, pp. 277.

[18] D. Choy, E. Gur-Esh, R. McVeigh, F. Müller, Content Management Interoperability Services (CMIS) Version 1.0, 2009, Retrieved November 9, 2009, from http://docs.oasisopen.org/cmis/CMIS/v1.0/cd04/cmis-spec-v1.0.pdf

[19] M-Y. Choi, E-A. Cho, D-H. Park, J-Y. Bae, C-J. Moon, D-K. Baik, A synchronization algorithm of mobile database for ubiquitous computing, in: Proceedings of the 2009 Fifth International Joint Conference on INC, IMS and IDC, IEEE Computer Society, Washington, DC, USA, 2009, pp. 416-419.

[20] Alfresco [Internet], Alfresco Document Management. Security, 2010, Available from: http://www.alfresco.com

[21] A. Mazen, M. Magid, M. Hemmasi, Lewis MF, In search of power: a statistical power analysis of contemporary research in strategic management, in: Acad Mgmt Proceed., 1985, pp. 30-34.

[22] L. Bardin, Análise de Conteúdo, Edições, Lisboa, 1977, pp. 70 (Portuguese).

[23] P. Horner, Mobile Technologies in EPICS-2: A Report Produced as Part of the North East Regional Collaboration for Personalised, Work-based, and Life-long learning (EPICS-2), Newcastle University, 2009, pp. 17.

[24] J. Moore, S. Oussena, P. Zhang, A portable document search engine to support off-line mobile learning, in: Proceedings of the IADIS International Conference Mobile Learning 2009, Spain, Barcelona, 2009.

[25] WebKit [Internet], WebKit Foundation, 2010, Available from: http://www.webkit.org

[26] R. Kay, Exploring the relationship between emotions and the acquisition of computer knowledge, Comput. Educ. 50 (May (4)) (2008) 1269-1283.

[27] E.W. Driessen, A.M.M. Muijtjens, J. Van Tartwijk, C.P. van der Vleuten, Web- or paper-based portfolios: is there a difference? Med. Educ. 41 (11) (2007) 1067-1073.

[28] B.M. Garrett, C. Jackson, A mobile clinical e-portfolio for nursing and medical students, using wireless personal digital assistants (PDAs), Nurse Educ. Pract. 6 (6) (2006) 339-346.

[29] S. Cotterill, P. Horner, M. Edney, North East Regional Collaboration for Personalised, Work-based, and Life-long Learning: EPICS-2, Sponsored by the Joint Information Systems Committee (JISC), Newcastle, UK, 2009.

[30] O.A. Alsos, A. Das, D. Svanæs, Mobile health IT: The effect of user interface and form factor on doctor-patient communication, Int. J. Med. Inf. 81 (January (1)) (2012) 12-28.

[31] M.J.W. Lee, A. Chan, Exploring the potential of podcasting to deliver mobile ubiquitous learning in higher education, $J$. Comput. High. Educ. 18 (Fall (1)) (2006) 94-115. 